\title{
Organ-specific enhancement of metastasis by spontaneous ploidy duplication and cell size enlargement
}

\author{
Xin $\mathrm{Lu}^{1}$, Xuemin $\mathrm{Lu}^{1}$, Yibin Kang ${ }^{1,2}$ \\ ${ }^{I}$ Department of Molecular Biology, Princeton University, Princeton, Washington Road, LTL 255, NJ 08544, USA, ${ }^{2}$ Breast Cancer \\ Program, The Cancer Institute of New Jersey, New Brunswick, NJ 08903, USA
}

\begin{abstract}
Aneuploidy is commonly observed in breast cancer and is associated with poor prognosis. One frequent type of aneuploidy, hypertetraploidy, may derive from ploidy duplication of hyperdiploid cells. However, the pathological consequences of ploidy duplication in breast cancer progression have not been characterized. Here, we present an experimental system demonstrating spontaneous appearance of hypertetraploid cells from organ-specific metastatic variants of the MDA-MB-231 breast cancer cell line through ploidy duplication in vitro and in vivo. The hypertetraploid progenies showed increased metastatic potential to lung and brain, but not to bone, which may be partially explained by the distinct capillary structures in these organs that confer differential lodging advantages to tumor cells with enlarged size. Our results suggest a potential mechanistic link between ploidy duplication and enhancement of metastatic potentials, as was observed in previous clinical studies of breast cancer.
\end{abstract}

Keywords: breast cancer; metastasis; organotropism; hyperploidy; cell size

Cell Research (2010) 20:1012-1022. doi:10.1038/cr.2010.93; published online 13 July 2010

\section{Introduction}

Malignant spread of cancer cells, or metastasis, accounts for the majority of cancer-related mortality [1, 2]. Better molecular and cellular understanding of the multistep metastasis cascade holds promise to control this dreadful disease. As one of the hallmarks of malignant cancer cells, aneuploidy is frequently observed in a large variety of cancer types and is associated with poor prognosis [3]. Several types of aneuploidy exist, including hypodiploidy, hyperdiploidy, hypertetraploidy and multiploidy. In breast adenocarcinoma, patients with hypertetraploid, hypodiploid and multiploid tumors had worse prognosis than those with diploid and hyperdiploid tumors [4], with hypertetraploid tumors being the most aggressive ones in some reports [5]. Hypertetraploidy was hypothesized to derive from endoreduplication of hyperdiploid cells during breast cancer progression [6] Alternatively, hypertetraploidy may arise as a conse-

Correspondence: Yibin Kang

Tel:+1-609-258-8834; Fax: +1-609-258-2340

E-mail: ykang@princeton.edu

Received 8 February 2010; revised 21 March 2010; accepted 6 April 2010; published online 13 July 2010 quence of cell fusion, which occurs in higher frequencies in inflamed tissues and tumors $[7,8]$. Hypertetraploid tumor cells typically have increased nuclear and cell size [9]. The increase of nuclear dimension during cancer progression has been shown to be associated with poor clinical outcomes in many different types of malignancies, such as breast cancer [10-12], prostate cancer [13, 14] and melanoma [15]. In particular, increased nuclear area was found to have strong correlations with advanced tumor stage, poor survival and increased risk of metastasis in breast cancer [10]. However, without a suitable experimental model system that recapitulates spontaneous formation of hypertetraploid tumor cells, the pathological consequences of hypertetraploidy remain poorly understood. Here, we present a model system for the spontaneous in vitro and in vivo derivation of hypertetraploid tumor cells from different metastatic variants of the MDA-MB-231 breast cancer cell line. We compared the cellular and molecular characteristics of hypertetraploid breast cancer cells with their hyperdiploid parental counterparts. Furthermore, through extensive evaluation of their in vivo metastatic behaviors, we linked the enhanced metastatic potential of hypertetraploid tumor cells to lung and brain with the specialized blood vessel structures in these organs. Our study established a model 
system to analyze the impact of spontaneous ploidy duplication on tumor progression and metastasis, and provided novel mechanistic insights for the organ-specific enhancement of metastatic abilities of hypertetraploid tumor cells.

\section{Results}

Spontaneous formation of hypertetraploidy from hyperdiploid tumor cells

Two organotropic metastatic variants of the MDAMB-231 human breast cancer cell line [2] - the lungmetastatic LM2 [16] and the bone-metastatic 1833 (renamed as BM1 in the current study to facilitate presentation) [17] - were passaged for nearly 1 year in vitro to evaluate the relative genomic and phenotypic stability of organ-specific metastatic cell lines. Weekly DNA content analysis revealed a surprising ploidy pattern shift for both lines (Figure 1A). Predominant $2 N / 4 N$ peaks ( $N=$ haploidy of parental MDA-MB-231) were gradually replaced by one peak slightly lower than $4 \mathrm{~N}$ and another peak close to $8 \mathrm{~N}$. Apparently, the original cell lines were repopulated by cells with approximately doubled DNA content. The three peaks were very obvious during the transition (that is, between weeks 21 and 25 in Figure 1A) with the middle peak (close to $4 \mathrm{~N}$ ) composed of the G2/M peak of the original (hyperdiploid) population and the G1/G0 peak of the newly evolved population (hyper- tetraploid). Such a mixed ploidy profile closely resembles the clinical observation of multiploidy in a subset of breast cancer patients [4], suggesting that multiploidy may be the transitional state between hyperdiploidy and hypertetraploidy. The genetic continuity of the two populations with distinct ploidy levels presented us with a unique opportunity to investigate the potential functional consequences of spontaneous ploidy duplication in malignant phenotypes.

To isolate the two closely matched and relatively pure subpopulations of hyperdiploid and hypertetraploid cells from the same cell line, we stained the cells at the multiploidy stage with Hoechst 33342 dye, and sorted the G1/ G0 peak of the hyperdiploid population (termed 'modal') and the $\mathrm{G} 2 / \mathrm{M}$ peak of the hypertetraploid population (termed 'hyper') (Figure 1B). Spectral karyotyping determined the average chromosome numbers for LM2-modal, LM2-hyper, BM1-modal and BM1-hyper to be 61, 103, 56 and 107, respectively (Figure 2A and 2B). The hyper cells adopted all major chromosomal aberrations of the parental modal cells, although a small number of new abnormalities also evolved (Figure 2C and 2D). Nearly doubled chromosomes and highly conserved chromosomal structures suggest that the hyper cells were indeed derived from the chromosomal complement duplication of the modal cells $[6,9]$, and ruled out the possibility that the appearance of the hypertetraploid population during long-term culture was due to the outgrowth of a con-
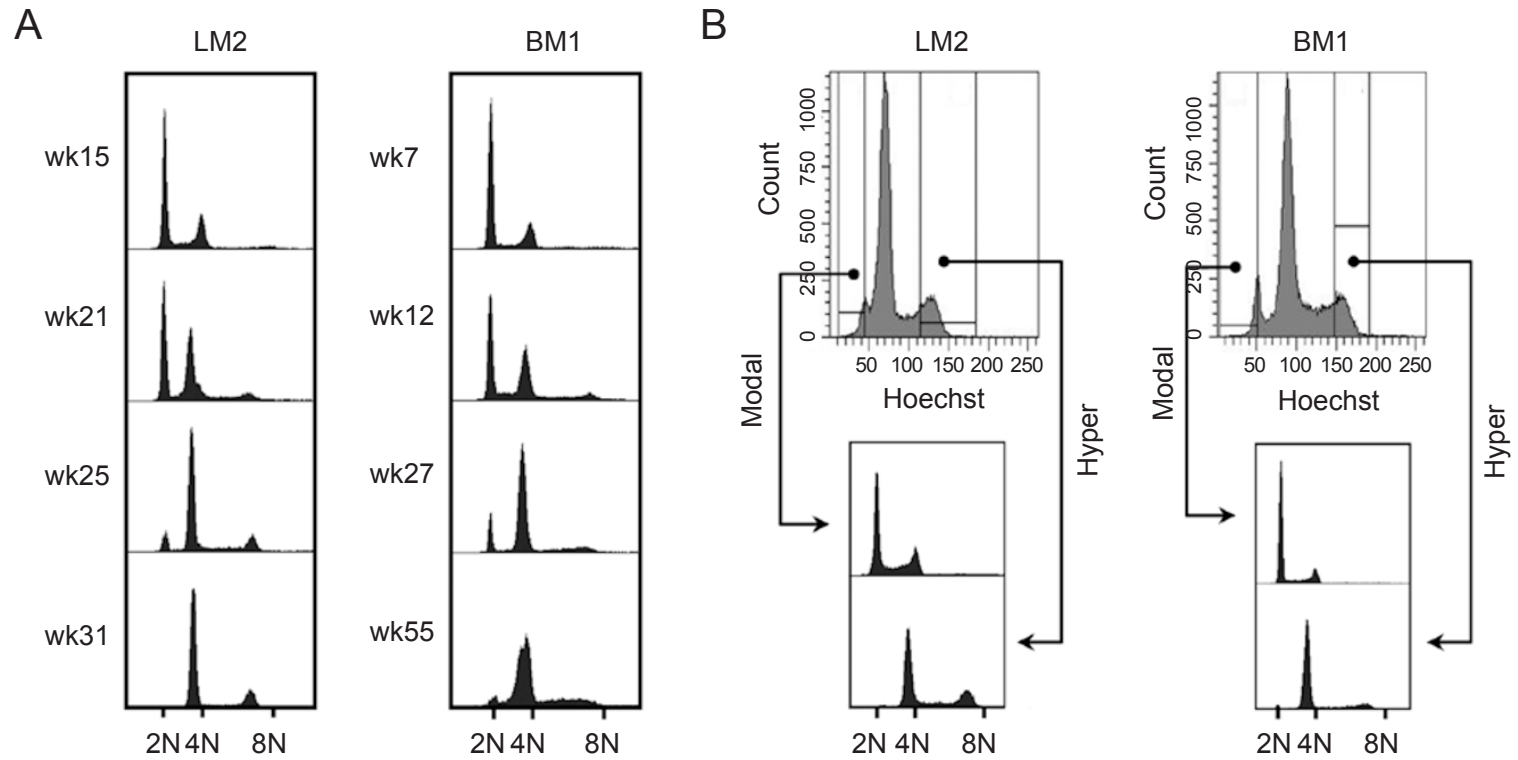

Figure 1 Spontaneous ploidy duplication generated hypertetraploid cells. (A) DNA content evolution of in vitro-cultured LM2 and BM1 during long-term cell culture. Flow cytometry DNA content profiles of cells collected at the indicated time point during long-term cell culture are shown. (B) Sorting of modal and hyper cells during the ploidy transition period after the cells were labeled with vital DNA dye Hoechst 33342. $N$ = haploidy of the parent MDA-MB-231 cell line. 


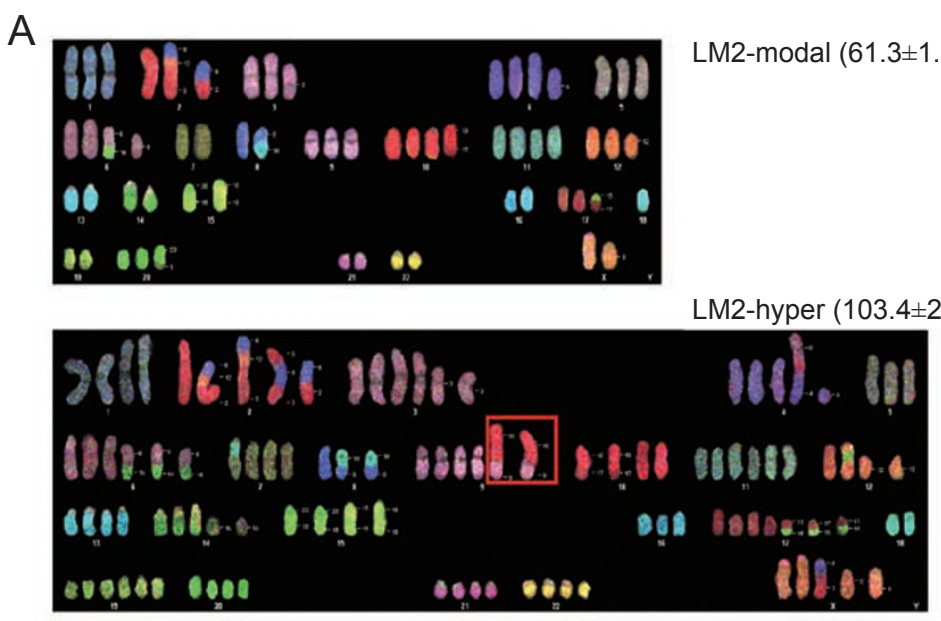

B
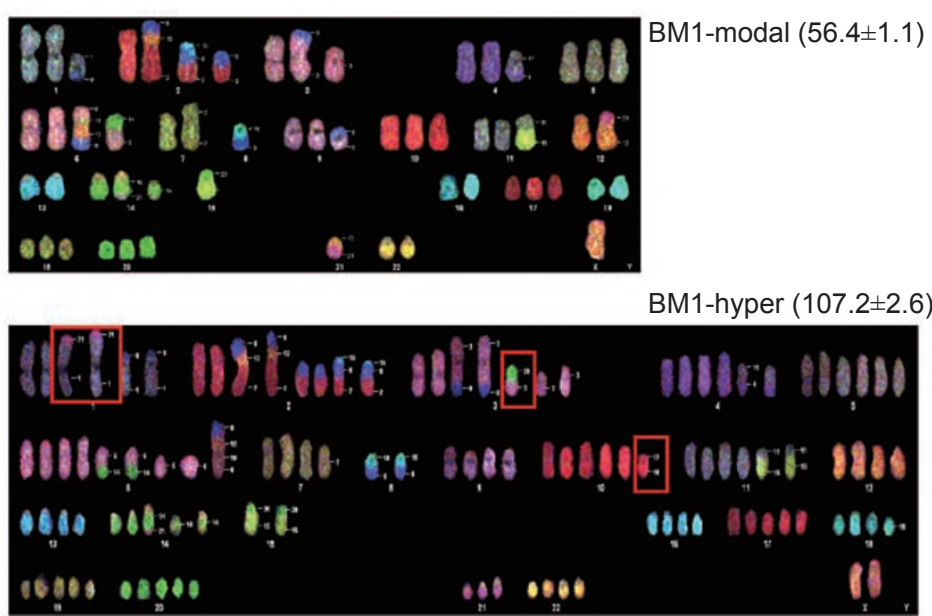

\begin{tabular}{|c|c|c|}
\hline Marker Chr. & $\begin{array}{l}\text { LM2- } \\
\text { modal }\end{array}$ & $\begin{array}{l}\text { LM2- } \\
\text { hyper }\end{array}$ \\
\hline $\begin{array}{c}\mathrm{t}(2 ; 8) \\
\mathrm{t}(2 ; 12 ; 8) \\
\operatorname{del}(3) \\
\text { del }(6) \\
\mathrm{t}(6 ; 14) \\
\mathrm{t}(8 ; 18) \\
\mathrm{t}(10 ; 17) \\
\text { del }(12) \\
\mathrm{t}(15 ; 15) \\
\mathrm{t}(15 ; 17) \\
\mathrm{t}(15 ; 20) \\
\mathrm{t}(17 ; 20) \\
\text { del }(\mathrm{X}) \\
\mathrm{t}(9 ; 10) \\
\text { del }(14) \\
\end{array}$ & $\begin{array}{l}+ \\
+ \\
+ \\
+ \\
+ \\
+ \\
+ \\
+ \\
+ \\
+ \\
+ \\
+ \\
+ \\
+ \\
- \\
\end{array}$ & $\begin{array}{l}+ \\
+ \\
+ \\
+ \\
+ \\
+ \\
+ \\
+ \\
+ \\
+ \\
+ \\
+ \\
+ \\
+ \\
+\end{array}$ \\
\hline Marker Chr. & $\begin{array}{l}\text { BM1- } \\
\text { modal }\end{array}$ & $\begin{array}{l}\text { BM1- } \\
\text { hyper }\end{array}$ \\
\hline $\mathrm{t}(1 ; 8)$ & + & + \\
\hline $\mathrm{t}(2 ; 8)$ & + & + \\
\hline $\begin{array}{l}\mathrm{t}(2 ; 8 ; 16) \\
\mathrm{t}(2 ; 12 ; 8)\end{array}$ & $\begin{array}{l}+ \\
+\end{array}$ & $\begin{array}{l}+ \\
+\end{array}$ \\
\hline $\operatorname{del}(3)$ & + & + \\
\hline $\mathrm{t}(3 ; 8)$ & + & + \\
\hline $\mathrm{t}(4 ; 11)$ & + & + \\
\hline $\mathrm{t}(6 ; 14)$ & + & + \\
\hline $\mathrm{t}(6 ; 19 ; 12 ; 8)$ & + & + \\
\hline$t(8 ; 18)$ & + & + \\
\hline $\mathrm{t}(11 ; 15)$ & + & + \\
\hline $\operatorname{del}(14)$ & + & + \\
\hline $\mathrm{t}(14 ; 21)$ & + & + \\
\hline $\mathrm{t}(15 ; 20)$ & + & + \\
\hline $\mathrm{t}(1 ; 21)$ & - & + \\
\hline $\mathrm{t}(3 ; 20)$ & - & + \\
\hline $\operatorname{del}(6)$ & - & + \\
\hline $\mathrm{t}(10 ; 17)$ & - & + \\
\hline$t(x ; 4)$ & - & + \\
\hline
\end{tabular}

Figure 2 Hypertetraploid (hyper) cells inherited the majority of the chromosomes with characteristic abnormalities (marker chromosomes) from the hyperdiploid (modal) cells. (A-B) Representative spectral karyotyping images showing the chromosomal composition of each cell line. Total chromosome number was indicated as average \pm s.d. with 20 metaphase spreads counted. Red squares indicate the newly evolved chromosome aberrations. (C-D) Summary of marker chromosomal translocations and deletions identified in the indicated cell lines.

taminated population distinct from MDA-MB-231. The proliferation rates of the hyper cells were nearly identical to the corresponding modal cells in culture (Figure 3A). The comparable growth rates and tumor characteristics were also observed in vivo (Figure $3 \mathrm{~B}$ and $3 \mathrm{C}$ ), indicating that ploidy change did not significantly change tumor proliferation, at least during the time window of the in vitro and in vivo tumor growth experiments.

Organ-specific promotion of metastasis by ploidy duplication

Since hypertetraploidy has been previously linked to poor clinical outcomes, we evaluated the potential contribution of ploidy duplication to metastasis development. Modal and hyper derivatives of LM2 and BM1 were subjected to in vivo lung metastasis and bone metastasis assays, respectively. We were also able to test brain metastasis, as LM2 cells showed mild-brain metastatic ability, consistent with the finding that genes mediating lung metastasis and brain metastasis partially overlapped [18]. All cells were labeled with a dual reporter expressing renilla luciferase and mRFP to facilitate bioluminescence imaging (BLI) and fluorescence imaging [19]. Intriguingly, differential metastatic behavior alterations were observed in different organs when hyper and modal cells were compared. In lung and brain, LM2-hyper caused significantly more metastasis burden than LM2-modal (Figure 4A-4D). The increased metastasis burden detected by BLI (Figure 4A and 4C) was further confirmed by necropsy and hematoxylin and eosin ( $\mathrm{H}$ and $\mathrm{E})$ stain- 
A

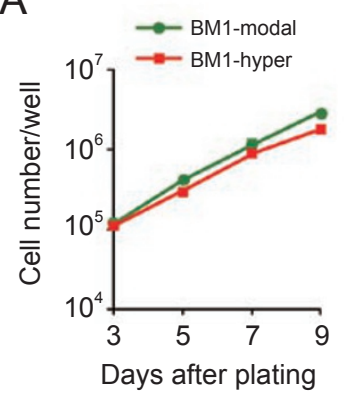

C

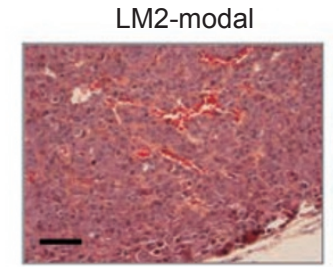

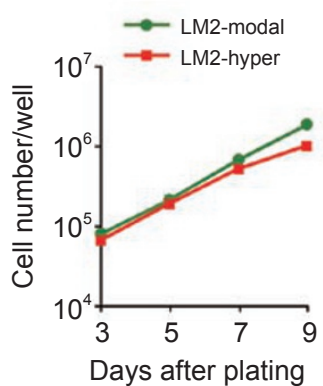

LM2-hyper

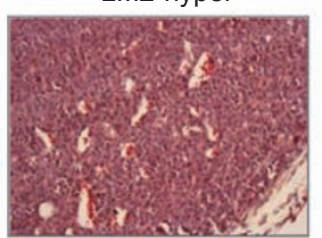

B
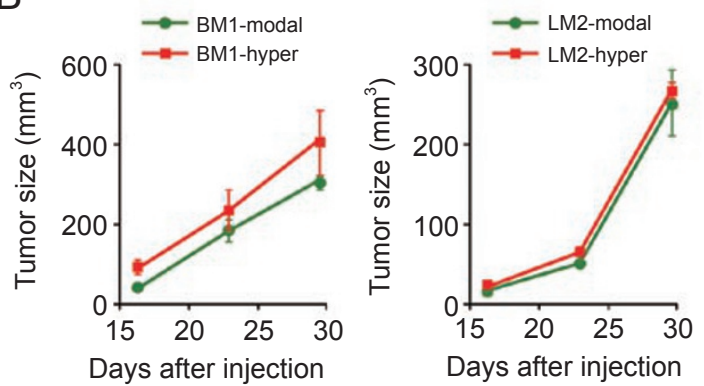

Days after injection

Figure 3 Similar growth rates of modal and hyper cells in vitro and in vivo. (A) In vitro growth curves showing similar growth rates of modal and hyper cells, for either BM1 or LM2, during the exponential growth phase. Data represent average \pm s.d. with triplicate for each line at each time point. (B) Tumor growth curves at the mammary glands with similar slopes across all time points, suggesting similar growth rates of modal and hyper cells, for both BM1 or LM2. Data represent average \pm s.e.m. $n=6$ tumors per group. (C) $\mathrm{H}$ and $\mathrm{E}$ staining of respective tumors. Scale bar represents $100 \mu \mathrm{m}$.

ing of metastatic lesions (Figure 4B and 4D). In contrast, BM1-modal and BM1-hyper showed no difference in colonizing the bone (Figure 4E). X-ray radiography and Goldner's trichrome staining showed similar degree of metastasis-associated osteolysis in the tibia (Figure 4F). The absence of enhanced metastatic potential to bone by BM1-hyper was not due to the optimal bone-metastatic ability that already exists in BM1-modal, because LM2hyper, derived from the weakly bone-metastatic LM2modal, also failed to display more metastatic potential to bone (data not shown).

DNA content analysis of freshly isolated metastases formed by LM2 or BM1 indicated that spontaneous ploidy duplication also occurred in vivo (Figure 5A and $5 \mathrm{~B})$, ruling out the possibility that the process was simply an in vitro artifact. Metastases formed by LM2-hyper and BM1-hyper showed stably maintained hypertetraploid DNA composition (Figure 5C and 5D), confirming that the phenotypic changes were caused by the ploidy doubling.

\section{Lack of transcriptomic changes due to ploidy duplication}

To explain the organ-specific enhancement of metastasis by ploidy duplication, we determined whether ploidy evolution differentially influenced the expression of genes important for organotropic metastasis. Previously identified bone-, lung- and brain-specific metas- tasis gene signatures using the MDA-MB-231 system [16-18] were used to cluster modal and hyper cells, as well as other MDA-MB-231 sublines with well-defined metastatic abilities [16-18] (Figure 6A-6C). Surprisingly, LM2-modal and LM2-hyper always clustered together, as is the case with BM1-modal and BM1-hyper. Ploidy duplication neither shifted the expression profile of a weakly metastatic cell to that of a highly metastatic one nor obviously changed the expression levels of signature genes for highly metastatic cells. These notions were further supported by gene set enrichment analysis [20, 21], which failed to find enrichment of either of the three signatures in hyper cells (see Materials and Methods for details). When LM2- and BM1-modal were compared with LM2- and BM-hyper to identify any ploidy-regulated gene(s), no such gene(s) could be found by statistical analysis (see Materials and Methods). Such findings may not be entirely unexpected if one considers the resistance of gene expression alteration to ploidy changes in yeast [22]. The absence of ploidy-regulated genes in our current study reflects the robustness of transcriptome when all (or most) genetic materials are synchronously duplicated.

\section{Enhanced organ-specific metastasis abilities linked to} cell size enlargement

Increased cell size is a conspicuous consequence of 
increased ploidy in yeast, plant and mammalian cells [9]. We observed an $\sim 1.5$-fold increase in cell volume of hyper cells (Figure 7A and 7B). Biophysical properties of circulating tumor cells, including adhesiveness, rigidity and size, were associated with metastatic ability in previous studies [23-25]. Therefore, we wanted to determine whether enlarged cell size by ploidy alteration might play any role in causing the organotropic enhancement of metastasis. Lodging of tumor cells within $12 \mathrm{~h}$ of inoculation in three organs were evaluated by fluorescence imag- ing of labeled tumor cells. In lung and brain, most tumor cells were within the capillaries, whereas in bone, most were located outside of vasculature (Figure 7C and 7D). Interestingly, there were significantly more LM2-hyper cells than LM2-modal cells arrested in the capillaries of lung and brain, whereas BM1-modal and BM1-hyper showed similar lodging efficiency in the bone marrow (Figure 7E). Recently, the structural feature of capillary walls in different organs was recognized as an important factor to affect tumor cell infiltration [26]. While the fe-
A

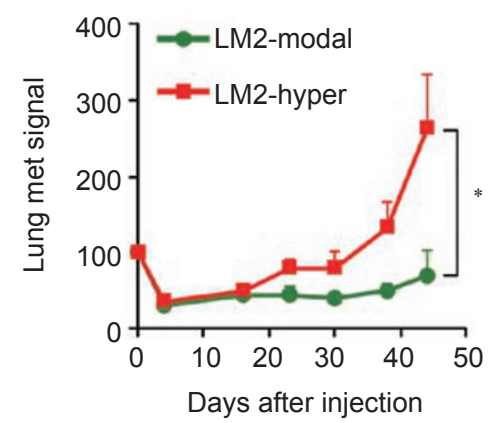

C

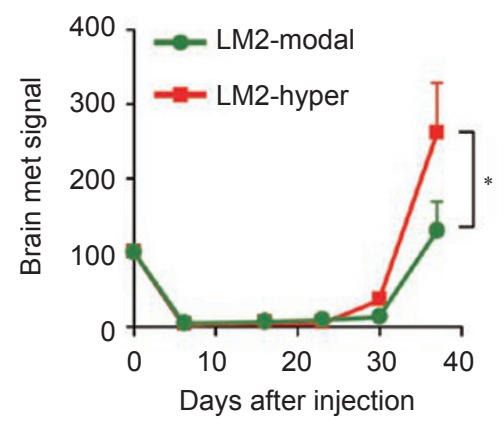

E

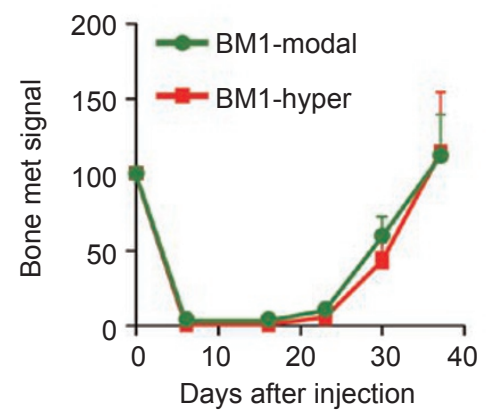

B
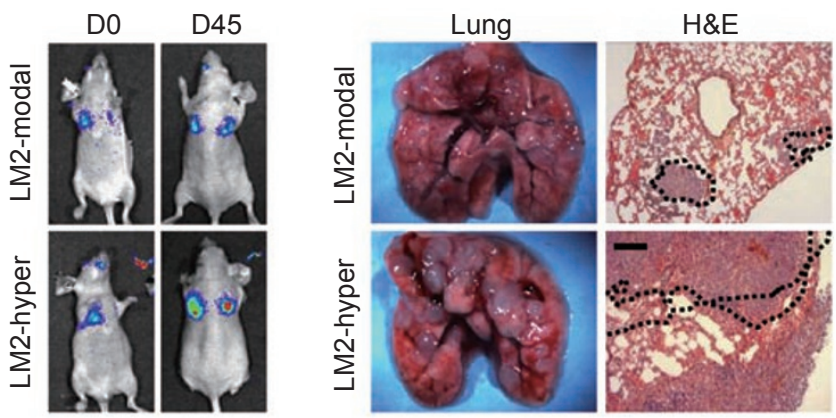

D
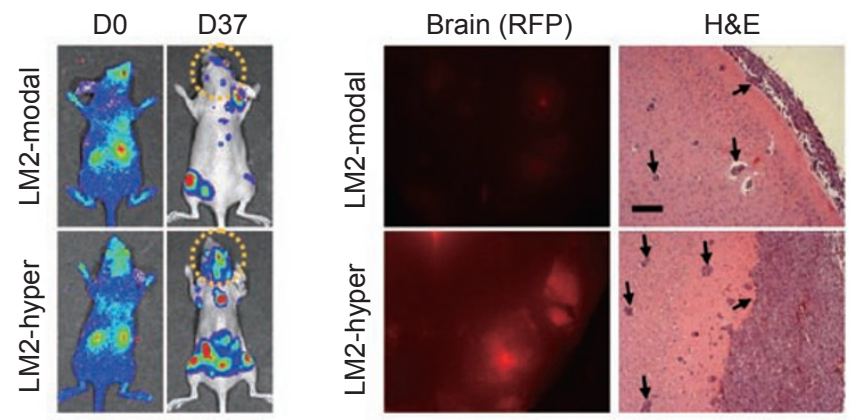

F
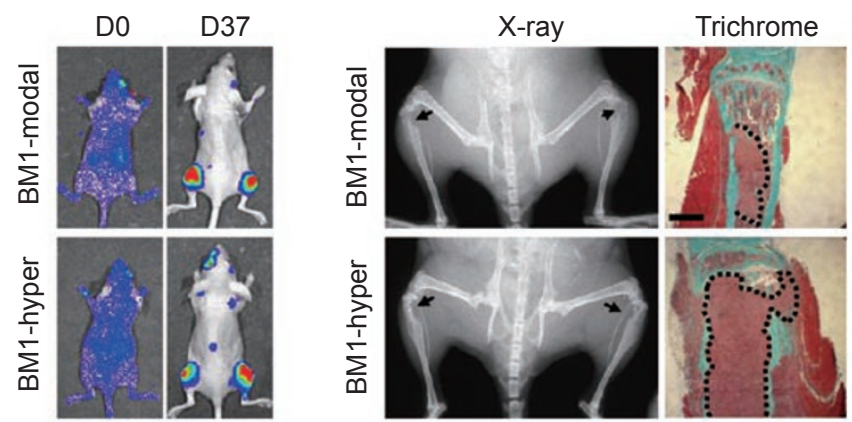

Figure 4 Ploidy duplication enhanced metastasis to lung and brain, but not to bone. (A) Metastasis burden quantified by bioluminescence imaging (BLI) at different time points after intravenous tumor cell injection showed that LM2-hyper was more metastatic to lung than LM2-modal, with representative mice, fresh lung tissue and $\mathrm{H}$ and $\mathrm{E}$ staining shown in B. (C) LM2-hyper was more metastatic to brain than LM2-modal after intracardiac injection, with representative mice, fresh brain tissue with fluorescent stereoscopy and $\mathrm{H}$ and $\mathrm{E}$ staining shown in D. (E) BM1-hyper and BM1-modal showed similar metastatic ability to bone after intracardiac injection, with representative mice, X-ray radiographs and Goldner's trichrome staining shown in $\mathbf{F}$. In $\mathbf{A}, \mathbf{C}$ and $\mathbf{E}$, error bar represents s.e.m. $n=8$ mice per group. $\left(^{*}\right) P<0.05$ with Mann-Whitney test. In $\mathbf{B}, \mathbf{D}$ and $\mathbf{F}$, major metastases were indicated by dotted contour line or arrow. Scale bars represent $200 \mu \mathrm{m}$ in $\mathbf{B}$ and $\mathbf{D}, 800 \mu \mathrm{m}$ in $\mathbf{F}$. 
A

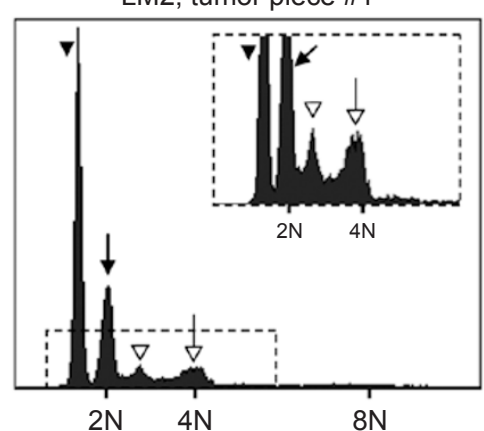

LM2, tumor piece \#2

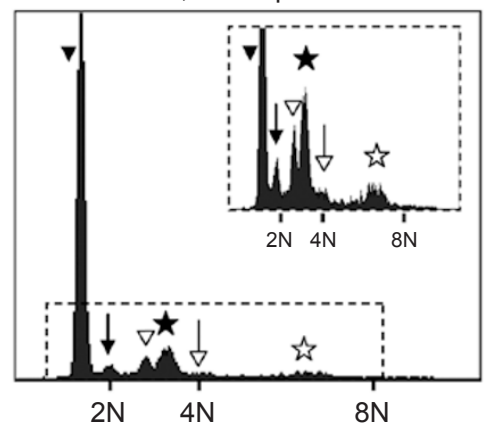

B

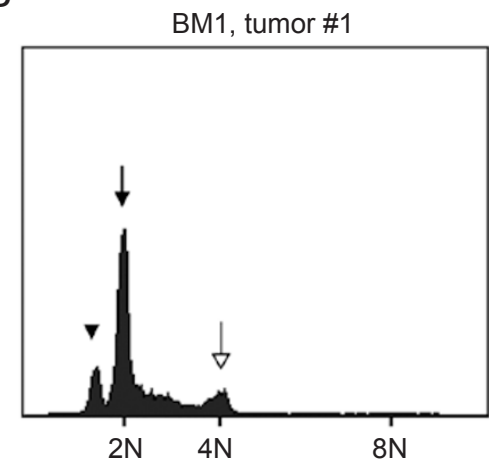

BM1, tumor \#2

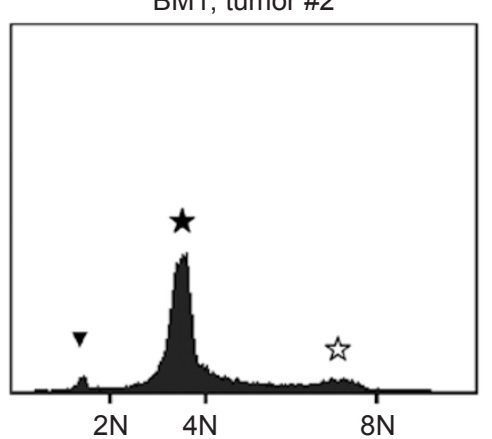

C

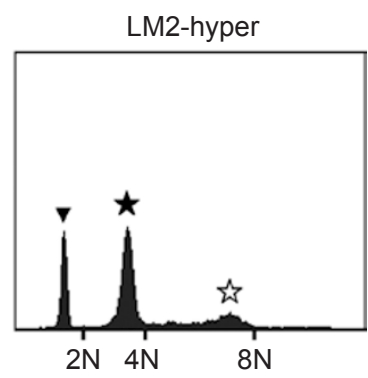

$\mathrm{D}$

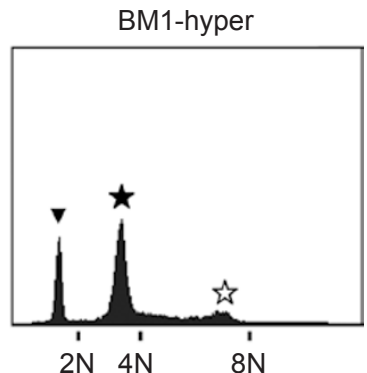

\begin{tabular}{|llllll|}
\hline$\downarrow$ Tumor modal G1 & $\star$ Tumor hyper G1 & v Mouse G1 & \\
$\downarrow$ & Tumor modal G2/M & 々 Tumor hyper G2/M & $\nabla$ & Mouse G2/M & N: tumor haploidy \\
\hline
\end{tabular}

Figure 5 Spontaneous ploidy duplication and stable DNA content of hypertetraploid cells during in vivo metastasis progression. (A) Two representative lung pieces with multiple metastatic nodules formed after intravenous injection of LM2 cells. Tumor no. 1 represents predominantly maintained hyperdiploidy of tumor cells in the nodules, while tumor no. 2 represents partially converted ploidy from hyperdiploidy to hypertetraploidy in the nodules One out of three lung pieces contained detectable hypertetraploid nodules. Inserts show the enlarged areas of the DNA content profiles. (B) Two representative bone marrow flushes with metastases formed after intracardiac injection of BM1 cells. Tumor no. 1 represents fully maintained hyperdiploidy of tumor cells, while tumor no. 2 represents almost completely converted ploidy from hyperdiploidy to hypertetraploidy (accounting for $18.2 \%$ of 11 tested bone samples). (C) Fully maintained hypertetraploidy of lung metastases formed by LM2hyper (accounting for $100 \%$ of four tested lung samples). (D) Fully maintained hypertetraploidy of bone metastases formed by BM1-hyper (accounting for $100 \%$ of two tested bone samples). Legend at the bottom shows signs representing different ploidy peaks of human or mouse origin. $N=$ haploidy of MDA-MB-231 cells.

nestrated structure of bone marrow sinusoid capillaries may be permissive to circulating tumor cells, the continuous endothelium in the lung and the tight blood-brain barrier in the brain could readily arrest tumor cells with a size larger than the capillary diameter, and only allow extravasation if the cells possess molecular properties that facilitate the penetration of vascular structures. Linking this finding to our current observations, we conclude that the enlarged cell size caused by ploidy duplication may result in more metastatic arrest of tumor cells only in the capillaries nonpermissive to free cell trafficking (that is, those in the lung and brain). In comparison, increase in cell size may not affect tumor cell infiltration through the fenestrated bone marrow capillaries.

\section{Discussion}

Hematogenous metastasis is a multistep process involving complex tumor-stroma interactions [1]. Most of the current efforts of metastasis research focus on the identification of genes and signaling pathways that mediate metastasis, with an emphasis on the organ-specific functions of the molecular mediators $[1,27]$. These efforts strived to understand the biochemical compatibility 
of tumor and stroma during metastasis and have successfully extended the concept of the 'seed' and 'soil' hypothesis into molecular levels [28]. However, another potentially important aspect of the 'seed' and 'soil' hypothesis, the biophysical compatibility between tumor cells and target organs, has been largely overlooked in the modern era of metastasis research. Systemic dissemination of tumor cell is not a random process. Instead, physical properties of tumor cells can influence the efficiency of the arrest and seeding in secondary organs. In the present study, by investigating the organ-specific metastasis behaviors of hypertetraploid cells and their isogenic hyperdiploid counterparts, we found that enlarged cell size confers a quantitative advantage when lodging at organs with continuous vasculature (that is, lung and brain), but not in an organ that has fenestrated capillaries (bone). This result is in line with a recent study suggesting that organ-specific vascular structures accounted for the se- lective advantages of TGF $\beta$-induced Angptl4 to promote lung metastasis but not bone metastasis [29]. The correlation between larger cell size with increased efficiency of metastatic seeding was also observed in a recent study of intravital real-time imaging of lung metastasis seeding and progression [30]. However, we could not rule out other possible mechanisms that could also account for the increased lung and brain metastasis by LM2-hyper cells. For example, hypertetraploid cells may be more resistant to rapid apoptosis after initial arrest compared with hyperdiploid cells in lung and brain, but not in bone, although the mechanism of organ specificity of apoptosis behavior changes by ploidy duplication is elusive.

It is obvious that enlarged cell size by ploidy duplication is not necessary for metastasis to occur; for example, LM2-modal is already highly metastatic to lung. BM1modal is essentially non-metastatic to lung [16]. If enlarged cell size were sufficient to confer metastasis abil-
A

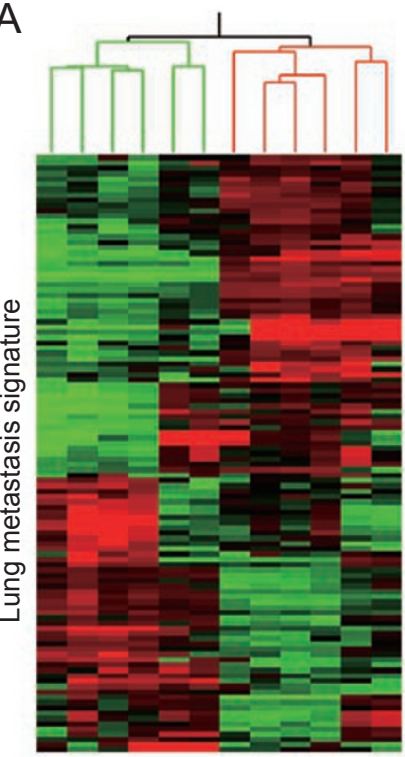

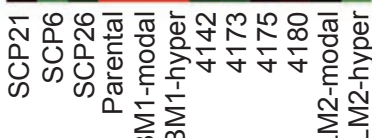
$\longleftarrow$ Weak $\longrightarrow$ ᄂ Strong $\lrcorner$
B

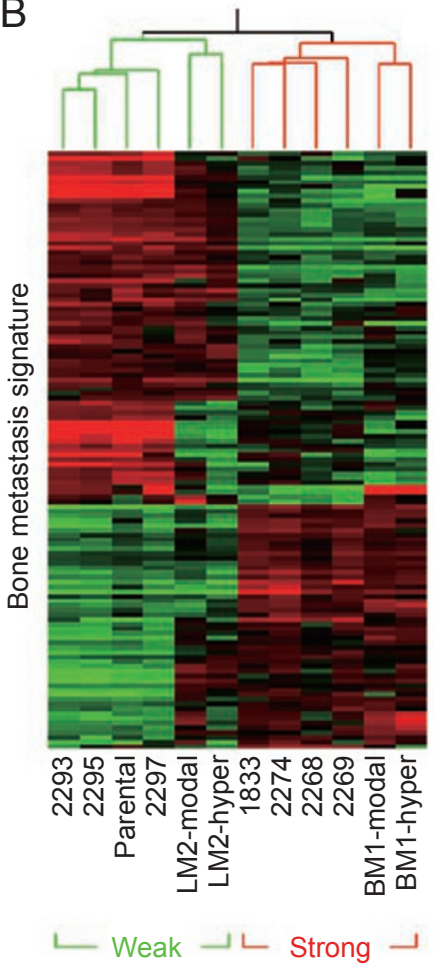

C

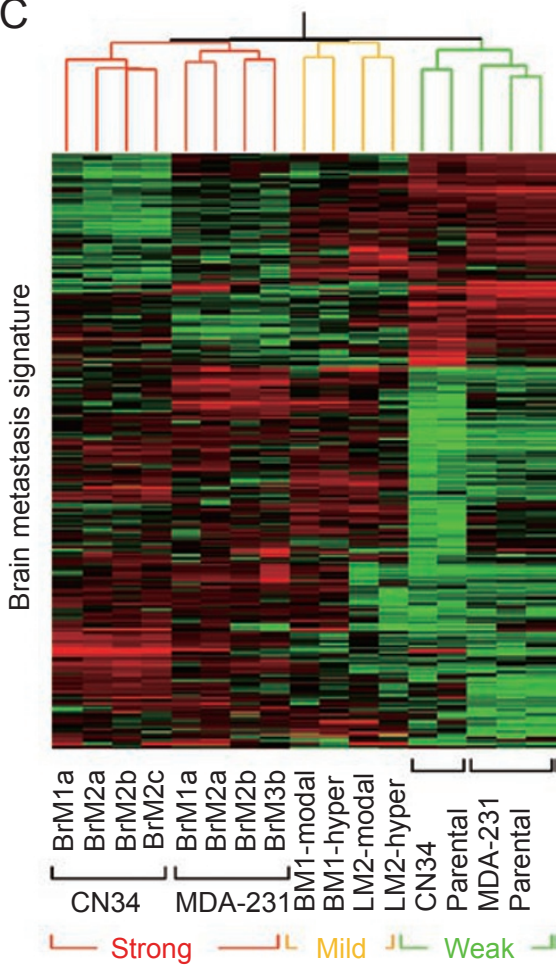

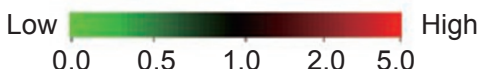

$\begin{array}{lllll}0.0 & 0.5 & 1.0 & 2.0 & 5.0\end{array}$

Figure 6 Modal and hyper cells maintain closely matched expression profile of organ-specific metastasis genes. (A) Lung metastasis signature [16] clustering BM1-modal and BM1-hyper together to the weakly lung-metastatic branch and LM2-modal and LM2-hyper together to the strongly lung-metastatic branch. (B) Bone metastasis signature [17] clustering LM2-modal and LM2-hyper together to the weakly bone-metastatic branch and BM1-modal and BM1-hyper together to the strongly bonemetastatic branch. (C) Genes correlated with brain metastatic behavior [18] clustering LM2-modal and LM2-hyper together, and BM1-modal and BM1-hyper together, to the mildly brain metastatic branch. In all dendrograms, colors code for metastatic ability: red, strong; orange, mild; green, weak. 
A

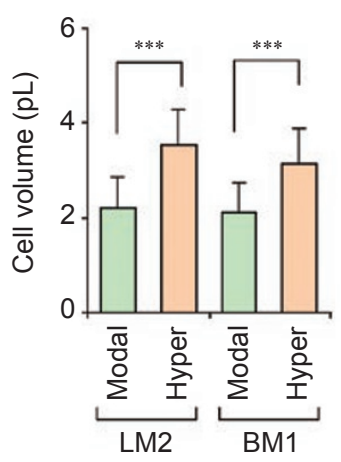

C
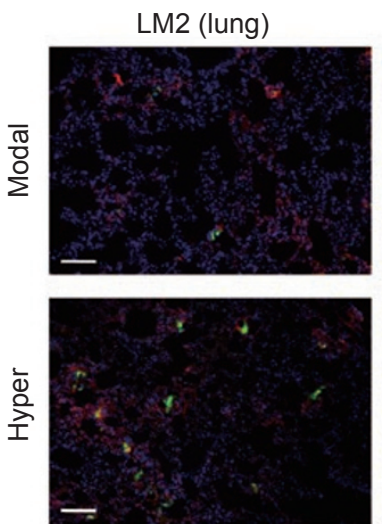

LM2 (brain)
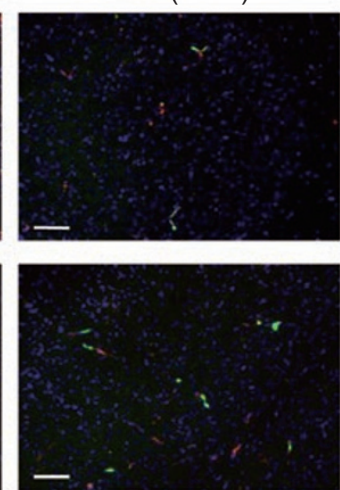

Blood vessel tumor cell DNA
B

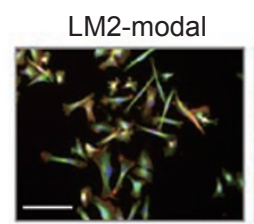

BM1-modal

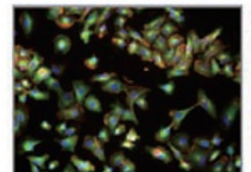

Actin $\alpha$-tubulin DNA
LM2-hyper

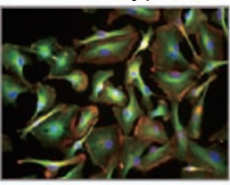

BM1-hyper

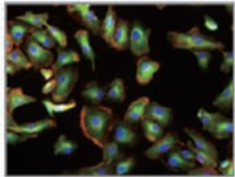

D
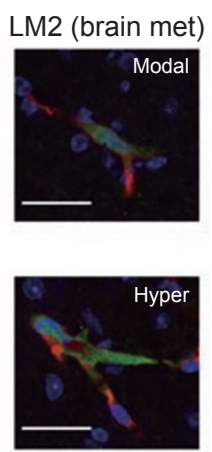

E

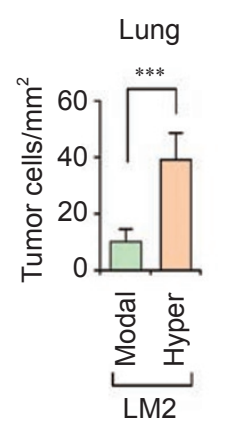

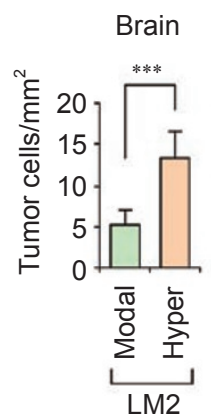

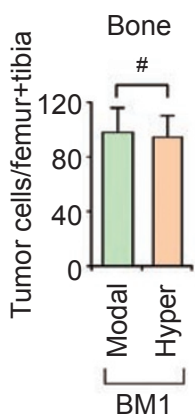

Figure 7 Enlarged cell size by ploidy duplication caused more cell arrest in the capillary beds in lung and brain, but not in bone. (A) Volume of cells in suspension measured with Coulter counter. Error bar represents s.e.m. of three independent measurements; *** $P<0.001$ with Student's $t$-test. (B) Cells in two-dimensional culture stained for actin with palloidin (red), $\alpha$-tubulin with antibody (green) and DNA with DAPI (blue). (C) Tumor cells (green; Cell tracer labeled) lodging in different organs within $24 \mathrm{~h}$ post-injection. Capillaries were stained with Texas Red dextran (red) and DNA with DAPI (blue). The few tumor cells in bone marrow were indicated by arrows. (D) High-resolution image of an LM2-modal cell or an LM2-hyper cell arrested in capillaries in the brain, with same color coding as in C. (E) Significantly increased tumor cell arrest in lung and brain and similar infiltration in bone. Error bar represents s.d. $n=4$ mice per group. *** $P<0.001$; \# $P>0.5$ with Student's $t$ test. Scale bar represents $100 \mu \mathrm{m}$ in B and C, $25 \mu \mathrm{m}$ in D.

ity to lung, we would expect BM1-hyper became lungmetastatic. Our preliminary experiments indicated that BM1-hyper, similar to BM1-modal, was still not able to form lung metastasis (data not shown). This finding indicates that the cell size increase, as a consequence of ploidy shift, is by itself not sufficient to enable lung metastasis formation. Taken together, the increased cell size only serves as a biophysical modulator of the metastasis ability. Gene expression pattern and biochemical activity of tumor cells still play a dominant role in determining the metastatic potential and organ specificity of malignant tumor cells. Indeed, the most likely reason for the inability of BM1-modal and BM1-hyper cells to colonize the lung is the lack of expression of essential genes for lung metastasis, such as ANGPTL4, EREG and COX2 [1,
16, 29].

The enlarged cell size is a consequence of ploidy duplication, as observed in our study. Ploidy duplication, or polyploidy in general, has profound influence during evolution [9], as well as cancer progression. Tetraploidy caused by cykokinesis failure promoted tumorigenesis [9] and was proposed as an intermediate stage for cancer aneuploidy during the early stages of tumorigenesis [9]. It should be noted that MDA-MB-231 is already aneuploid before any experimental handling. The ploidy shift studied here was the transition from hyperdiploidy (one type of aneuploidy) to hypertetraploidy (another type of aneuploidy). Ploidy duplication may lead to chromosome reshuffling and massive instability. However, our observation indicated that the hypertetraploid 
descendants were genetically stable, consistent with our previously reported finding that tumor-tumor cell fusion in MDA-MB-231 generated stable hybrids [19]. The genetic stability is possibly linked to the ability of supernumerary centrosomes to cluster into two functional mitotic spindles in advanced malignant cells [19]. The similarity of genomic stability between hypertetraploid cells and cells derived from fusion of MDA-MB-231 cells [19] raises the possibility that cell fusion may initiate ploidy duplication. Alternatively, endoreduplication as a result of cytokinesis failure or mitotic slippage can also lead to chromosome doubling [31]. During the course of our study, we observed that not all MDA-MB231-derived cells were able to spontaneously progress to higher ploidy after extensive period of cell culture. Another MDA-MB-231 subline, SCP2, did not progress to higher ploidy when cultured for nearly 1 year. Since both SCP2 and LM2 undergo spontaneous cell fusion at a low frequency [19], yet only LM2 could undergo massive ploidy duplication, endoreduplication instead of cell fusion is more likely to be the cause of ploidy shift in our current experimental system. Nevertheless, the exact cellular and molecular mechanism of ploidy duplication remains obscure and should be further investigated.

Overall, results from this study suggest that metastasis is not simply a metastasis gene-driven event but also a process subjected to influence by the biophysical properties of the constantly evolving tumor cells. Importantly, our study provided a direct mechanistic explanation for the previous clinical observations that hypertetraploidy and enlarged nuclear size are linked to poor survival and metastasis [5, 10-12]. In addition, the model system established in our study may become a useful platform for functional studies of putative molecular mediators of ploidy duplication, which could be explored as potential new targets for prophylactic cancer therapy to prevent metastasis. Further efforts should investigate the generality of these findings in breast as well as other types of cancer and explore the clinical association of hyperploidy with organ-specific metastases.

\section{Materials and Methods}

\section{Cell culture}

LM2 (4175) [16], BM1 (1833) [19] and their sublines were maintained in DMEM with10\% FBS and antibiotics.

Flow cytometry, spectral karyotyping and cell volume measurement

DNA content analysis and sorting with propidium iodide staining, Hoechst 33342 staining and subsequent flow cytometry were performed as described [19]. Spectral karyotyping was performed by the SKY/FISH facility in the Roswell Park Cancer Institute as described [19]. Cell volume was measured with a Coulter counter as described [19].

\section{In vitro growth curve}

Cells $\left(2 \times 10^{4}\right)$ were seeded into six-well plates with three wells/cell line/time point. Medium was changed every 2 days. Cells were counted every 2 days.

\section{Tumor xenografts and analysis}

All procedures involving mice, such as housing and care, and all experimental protocols were approved by the institutional animal care and use committee of Princeton University. For intracardiac injections to generate bone and brain metastases, $10^{5}$ cells in PBS were injected into the left cardiac ventricle of 4-week-old female nude mice (NCI) as described [17, 18, 32]. For intravenous injection to generate lung metastases, $2 \times 10^{5}$ cells in PBS were injected into the tail vein of nude mice, as described [16]. Development of metastases in bone and lung was monitored by BLI with the IVIS Imaging System (Xenogen) as described [16, 17]. BLI analysis was performed with Living Image software (Xenogen) by measuring photon flux of the region of interest. X-ray radiography analysis of bone lesions was performed using procedures as described [17]. For the orthotopic xenograft model, mammary fat pad injections and tumor size measurements were performed as described $[16,32]$.

\section{Histological and fluorescence analyses}

Hindlimb bone, lung and brain were excised, fixed in $10 \%$ neutral-buffered formalin, decalcified (for bone only) and embedded in paraffin for $\mathrm{H}$ and $\mathrm{E}$ staining or Goldner's trichromse staining [33]. Immunofluorescent staining of microtubules was performed with mouse anti- $\alpha$-tubulin (Sigma) primary antibody and Alexa Fluor 568-conjugated secondary antibody. Actin was stained with Alexa Fluor 488-conjugated phalloidin. To study the tumor cell arrest in different organs, tumor cells were labeled with the greenfluorescent vital dye CFDA SE Cell Tracer (Invitrogen) following the manufacturer's instruction. The inherent mRFP label was lost during the tissue fixation step, and therefore could not be used for tracing the cells. Tumor cells were injected into nude mice. After $12 \mathrm{~h}$, mice were injected with $1 \mathrm{mg}$ per mouse of Texas Redconjugated dextran (70 $000 \mathrm{MW}$, Invitrogen) $10 \mathrm{~min}$ before killing to label blood vessels. Lung, brain and bone were fixed with cold $10 \%$ formalin, decalcified (for bone only) and embedded in OCT for frozen sectioning (10 $\mu \mathrm{m}$ per section) and fluorescence imaging. Tumor cells were quantified as number of cells per field for lung and brain and converted to number of cells per $\mathrm{mm}^{2}$. For bone, because tumor cells were sparse, they were expressed as total number of cells per hindlimb (femur and tibia) based on calculations of 20 sections (200 $\mu \mathrm{m}$ in total) and the thickness of the bone cavity $(\sim 1 \mathrm{~mm})$.

\section{DNA content analysis of primary metastasis lesions from mice}

Small pieces of lung with multiple metastasis nodules were minced and digested as described [34]. Femur or tibia with metastatic lesions was flushed as described [35]. Red blood cells were lysed with ACK lysis buffer [36]. Cells were fixed immediately or briefly cultured overnight before fixation and subjected to DNA content analysis as described [19]. 


\section{Microarray analysis}

The same procedure as previously described [17] was used to conduct the microarray experiments for LM2-modal, LM2-hyper, BM1-modal and BM1-hyper on the Affymetrix U133A chips. Data were deposited at the NCBI Gene Expression Omnibus (http:// www.ncbi.nlm.nih.gov/geo/) with the accession no. GSE16554. Hierarchical clustering was performed with the Genespring GX 7.3 software (Agilent Technologies) using the organ-specific metastasis gene signatures and associated cell line microarray data [16-18]. To identify ploidy-regulated gene(s), four cell lines were separated into the modal group containing LM2- and BM1-modal and the hyper group containing LM2-and BM1-hyper. ANOVA was used to find genes with significant difference between two groups with Genespring GX 7.3 software (Agilent Technologies) using following conditions: pamametric test do not assume equal variance; FDR 0.05; multiple testing correction Benjamini and Hochberg false discovery rate (FDR). GSEA software [20, 37] was used to analyze the enrichment for the following gene sets: upregulated and downregulated subsets of the lung metastasis signature [16], upregulated and downregulated subsets of the bone metastasis signature [17], upregulated and downregulated subsets of the genes associated with brain metastatic behavior [18]. FDR cutoff of 0.25 was used as criterion of statistical significance as recommended.

\section{Statistical analysis}

Results were reported as average \pm s.d. (standard deviation) or s.e.m. (standard error of the mean), indicated in the figure legends. Comparisons were performed using unpaired two-sided Student's $t$-test without equal variance assumption or nonparametric MannWhitney test.

\section{Competing financial interests}

The authors declare no competing financial interests.

\section{Acknowledgments}

We thank M Yuan and M Bisher for assistance with histology; C DeCoste for assistance with flow cytometry. YK is a Champalimaud Investigator and a Department of Defense Era of Hope Scholar Award recipient. This research was additionally supported by grants from the National Institutes of Health (R01CA134519), the American Cancer Society, the Susan G Komen for the Cure and the New Jersey Commission on Cancer Research. Xin $\mathrm{Lu}$ is a recipient of a Harold W Dodds Fellowship from the Princeton University.

\section{References}

1 Gupta GP, Massague J. Cancer metastasis: building a framework. Cell 2006; 127:679-695.

2 Lu X, Kang Y. Organotropism of breast cancer metastasis. $J$ Mammary Gland Biol Neoplasia 2007; 12:153-162.

3 Rajagopalan H, Lengauer C. Aneuploidy and cancer. Nature 2004; 432:338-341.

4 Coulson P, Thornthwaite J, Woolley T, Sugarbaker E, Seckinger D. Prognostic indicators including DNA histogram type, receptor content, and staging related to human breast cancer patient survival. Cancer Res 1984; 44:4187-4196.
5 Kallioniemi OP, Blanco G, Alavaikko M, et al. Improving the prognostic value of DNA flow cytometry in breast cancer by combining DNA index and S-phase fraction. A proposed classification of DNA histograms in breast cancer. Cancer 1988; 62:2183-2190.

6 Dutrillaux B, Gerbault-Seureau M, Remvikos Y, Zafrani B, Prieur M. Breast cancer genetic evolution: I. Data from cytogenetics and DNA content. Breast Cancer Res Treat 1991; 19:245-255.

7 Lu X, Kang Y. Cell fusion as a hidden force in tumor progression. Cancer Res 2009; 69:8536-8539.

8 Singec I, Snyder EY. Inflammation as a matchmaker: revisiting cell fusion. Nat Cell Biol 2008; 10:503-505.

9 Storchova Z, Pellman D. From polyploidy to aneuploidy, genome instability and cancer. Nat Rev Mol Cell Biol 2004; 5:45-54.

10 Abdalla F, Boder J, Markus R, Hashmi H, Buhmeida A, Collan Y. Correlation of nuclear morphometry of breast cancer in histological sections with clinicopathological features and prognosis. Anticancer Res 2009; 29:1771-1776.

11 van Bogaert LJ, de Muylder C, Maldague P, Maisin H. Prognostic implications of mean nuclear diameter in breast cancer. Br J Cancer 1980; 42:537-541.

12 Zajdela A, De LaRiva LS, Ghossein NA. The relation of prognosis to the nuclear diameter of breast cancer cells obtained by cytologic aspiration. Acta Cytologica 1979; 23:75-80.

13 Khan MA, Walsh PC, Miller MC, et al. Quantitative alterations in nuclear structure predict prostate carcinoma distant metastasis and death in men with biochemical recurrence after radical prostatectomy. Cancer 2003; 98:2583-2591.

14 Blom JH, Ten Kate FJ, Schroeder FH, van der Heul RO. Morphometrically estimated variation in nuclear size. A useful tool in grading prostatic cancer. Urol Res 1990; 18:93-99.

15 Mossbacher U, Knollmayer S, Binder M, Steiner A, Wolff K, Pehamberger $\mathrm{H}$. Increased nuclear volume in metastasizing ‘thick' melanomas. J Invest Dermatol 1996; 106:437-440.

16 Minn AJ, Gupta GP, Siegel PM, et al. Genes that mediate breast cancer metastasis to lung. Nature 2005; 436:518-524.

17 Kang Y, Siegel PM, Shu W, et al. A multigenic program mediating breast cancer metastasis to bone. Cancer Cell 2003; 3:537-549.

18 Bos PD, Zhang XHF, Nadal C, et al. Genes that mediate breast cancer metastasis to the brain. Nature 2009; 459:10051009.

19 Lu X, Kang Y. Efficient acquisition of dual metastasis organotropism to bone and lung through stable spontaneous fusion between MDA-MB-231 variants. Proc Natl Acad Sci USA 2009; 106:9385-9390.

20 Subramanian A, Tamayo P, Mootha VK, et al. Gene set enrichment analysis: a knowledge-based approach for interpreting genome-wide expression profiles. Proc Natl Acad Sci USA 2005; 102:15545-15550.

21 Mootha VK, Lindgren CM, Eriksson KF, et al. PGC-1alpharesponsive genes involved in oxidative phosphorylation are coordinately downregulated in human diabetes. Nat Genet 2003; 34:267-273.

22 Galitski T, Saldanha AJ, Styles CA, Lander ES, Fink GR. Ploidy regulation of gene expression. Science 1999; 285:251254. 
23 Watanabe S. The metastasizability of tumor cells. Cancer 1954; 7:215-223.

24 Zeidman I. The fate of circulating tumor cells: I. Passage of cells through capillaries. Cancer Res 1961; 21:38-39.

25 Koike A. Mechanism of blood-borne metastases. I. Some factors affecting lodgment and growth of tumor cells in the lungs. Cancer 1964; 17:450-460.

26 Nguyen DX, Bos PD, Massague J. Metastasis: from dissemination to organ-specific colonization. Nat Rev Cancer 2009; 9:274-284.

27 Kang Y. Functional genomic analysis of cancer metastasis: biologic insights and clinical implications. Expert Rev Mol Diagn 2005; 5:385-395.

28 Paget S. Distribution of secondary growths in cancer of the breast. Lancet 1889; 1:571-573.

29 Padua D, Zhang XH, Wang Q, et al. TGFbeta primes breast tumors for lung metastasis seeding through angiopoietin-like 4. Cell 2008; 133:66-77.

30 Kimura H, Hayashi K, Yamauchi K, et al. Real-time imaging of single cancer-cell dynamics of lung metastasis. J Cell Biochem 2010; 109:58-64.
31 Ganem NJ, Storchova Z, Pellman D. Tetraploidy, aneuploidy and cancer. Curr Opin Genet Dev 2007; 17:157-162.

32 Kang Y. Analysis of cancer stem cell metastasis in xenograft animal models. Methods Mol Biol 2009; 568:7-19.

33 Lu X, Kang Y. Chemokine (C-C motif) ligand 2 engages CCR2+ stromal cells of monocytic origin to promote breast cancer metastasis to lung and bone. J Biol Chem 2009; 284:29087-29096.

34 Yang J, Mani SA, Donaher JL, et al. Twist, a master regulator of morphogenesis, plays an essential role in tumor metastasis. Cell 2004; 117:927-939.

$35 \mathrm{Lu}$ X, Wang Q, Hu G, et al. ADAMTS1 and MMP1 proteolytically engage EGF-like ligands in an osteolytic signaling cascade for bone metastasis. Genes Dev 2009; 23:1882-1894.

36 Sauer KA, Scholtes P, Karwot R, Finotto S. Isolation of CD4+ T cells from murine lungs: a method to analyze ongoing immune responses in the lung. Nat Protoc 2007; 1:2870-2875.

37 Mootha VK, Lindgren CM, Eriksson K-F, et al. PGC1 [alpha]-responsive genes involved in oxidative phosphorylation are coordinately downregulated in human diabetes. Nat Genet 2003; 34:267-273. 